\title{
Research of Network Teaching Platform Based Grid
}

\author{
${ }^{\mathrm{a}}$ Zhang Shunli, ${ }^{\mathrm{b}}$ Yin Qingshuang \\ ${ }^{a, b}$ The Department of Computer Science and Engineering Henan Institute of Engineering Zhengzhou,China
}

\begin{abstract}
As an emerging technology, grid combines and integrates the whole Internet resources to form a supercomputer, allowing the complete sharing of resources. Applying grid to network teaching can eliminate and solve the disadvantages and problems which exist in traditional network teaching. This paper briefly introduces the grid technology and its application in network teaching. It talks about the principle of network teaching platform based grid, design and analysis modules.
\end{abstract}

Index Terms: grid; open grid services architecture; network teaching; teaching platform

(C) 2012 Published by MECS Publisher. Selection and/or peer review under responsibility of the International Conference on E-Business System and Education Technology

\section{Introduction}

With the increasing development of technology, network teaching plays an important role in the education field, which greatly extends time and space with its advanced technology. This has changed the relationship between teaching and learning, providing a faster and easier way of learning, enhancing the student's interest, efficiency and initiative. However, because of the drawbacks of the Internet itself from heterogeneity of hardware and software systems, the development of network teaching meets some obstacles, such as the lack of extensive resource sharing, the development of repeating learning resources seriously, effecting collaboration between students and teachers. Applying grid technology to the teaching network will greatly change the status quo and effectively solve the problems which hinder network teaching development.

\section{The overview of grid technology}

Grid is an emerging technology that is in constant development and change as the third wave following the tradition of the Internet, and can also be called the third generation of Internet. The grid is a network infrastructure in information society, which connects all resources on the Internet to achieve interoperability, including computing, storage, communication and software resources and so on [1]. 


\subsection{The Technical Characteristics of Grid}

Grid system is a seamless integrated environment of computing, collaborating with the following characteristics [1]:

\section{1) Resource sharing}

The sharing of resources is a fundamental characteristic of the grid. Grid technology can realize all network resource sharing including various resources. A major purpose of the Grid is to eliminate the resources isolated island so as to completely share resources across Internet.

\section{2) Dynamic}

The grid has excellent dynamic. For a grid system its size, service and resources are constantly changing. The grid technology can optimize all the resource allocated by continually updating educational resources to ensure the latest information to users.

\section{3) Heterogeneity}

Grid can connect various heterogeneous network systems and the most extensive and comprehensive education resources. In grid environment, users can easily and quickly share and exchange the resources regardless of the platform being used.

\section{4) Supercomputing performance}

The grid system can combine all computers together which are distributed in different geographic locations to become a virtual supercomputer with supercomputing performance. Virtual laboratory, space and Teleimmersion network education services all need strong calculating ability, which is very difficult to realize in the traditional network environment, while being easily obtained in grid environment.

\section{5) Strong support services}

Grid can provide accurate service intelligently and quickly according to the needs of learners. When users input their own requests in grid system, the resources needed can be located and returned to them quickly and accurately [2].

\subsection{Open Grid Services Architecture}

Grid system architecture explains the construction of the grid system, which includes the definition and functional description of grid basic components, the relationship and inheriting method of all parts, and effective operation mechanism of grid system [2]. For the moment, the most influential grid system architecture is Open Grid Services Architecture (OGSA).

OGSA is a service-oriented concept. It may support temporary services and be able to realize the dynamic creation and deletion of said services [1]. Grid services include many various interfaces, but Grid Services interface is indispensable.

OGSA can be abstracted as three layers, they are: from bottom to top, they are shown in Fig. 1:

\section{1) Resource layer (including physical and logical resources)}




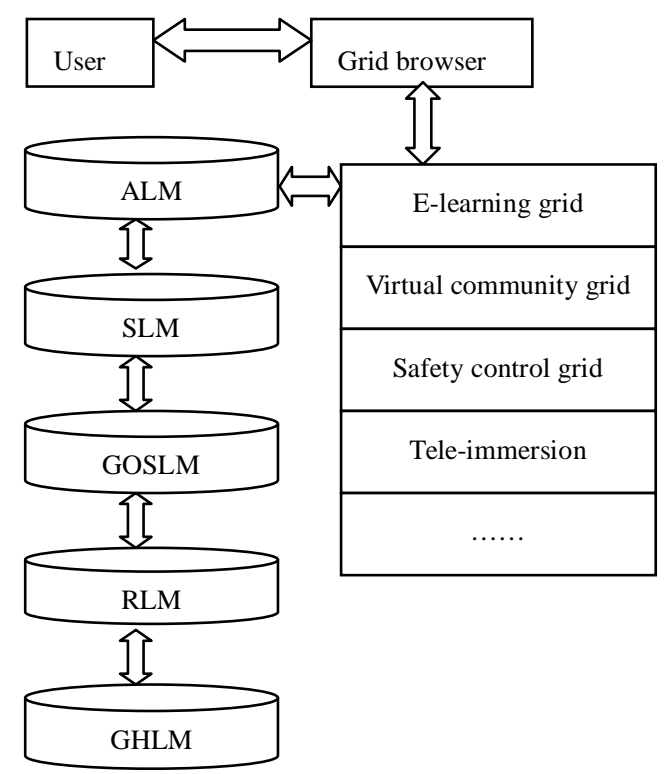

Figure 1. The structure model of OGSA

Physical resources include servers, storage and network. Logical resources with physical resource above it provide extra functionality. Through Grid Middleware, they provide numerous abstract services for the layer above them.

\section{2) Service layer (middleware layer and development tools layer)}

OGSA defines the grid services for all resources by defining specified standard interfaces, behaviors and interactive methods [1]. Middleware layer is a series of tools and protocol software, also called Grid Operating System. Its function is to provide programming interfaces and the corresponding environment for users.

\section{3) Application layer}

Application layer is the embodiment of users demand. By using the tools and environment provided by grid operating system, users can easily develop all the application systems.

\section{The application of grid in network teaching}

The application of grid in network teaching mainly exists in the following three areas:

\section{1 Building a Good Learning Environment for Collaborative Learning}

The grid builds up a good platform for collaborative learning, which is very helpful in cultivating students learning and advanced coordination ability in the teaching process [3].

\section{2 Implementing Education Resources Sharing}

The biggest characteristic of grid technology is to realize sharing all resources in the grid environment [4]. Through grid technology, users can accurately and rapidly locate various types of learning resources needed to enhance their learning efficiency. 


\section{3 Realizing the Personalized Service}

The main bottleneck of individualized service network is how to solve the problem of network bandwidth [5]. Making full use of the characteristics of grid technology can reduce the dependence on network bandwidth and effectively achieve the personalized service.

From the above analysis of grid application in network teaching, it can be observed that the teaching network based on grid eliminates the problems which exist in the traditional network teaching method, and has incomparable advantages compared with traditional network teaching, and truly realizes real resource sharing and personalized service and collaborative learning. Therefore, in my opinion establishing the network teaching system based on grid is imperative.

\section{The design principle of network teaching platform based grid}

As a new platform of network teaching, grid provides a supporting mechanism of using various resources for network teaching by connecting and integrating all kinds of resources into a virtual computing system [6]. The main design principles are as follows:

\section{1 Powerful Management Functions of Teaching Resources and Resource Utilization Efficiency}

In order to improve the student learning efficiency online and the teacher's leading role, the platform needs to provide a powerful teaching resource management function, to optimize resource allocation adapting to the needs of student autonomy and cooperative learning.

The platform should ensure users can make full use of the idle resources in the gird teaching system to calculate backup or restore data to improve the utilization efficiency of resources.

\section{2 Transparency and Intelligence Services}

Platform should be able to provide the single entry which is easy for users to access all kinds of resources transparently [7]. Although these resources are stored in different locations for visitors, through the grid browser users can enjoy various resources the platform provides without prior knowledge of platform structure and working principle.

By application of grid technology, the platform should provide a higher level of personalized service, which allows users to access the resources they need in the shortest possible time.

\section{3 Scalability and Flexibility}

Good expansibility and flexibility can ensure users can add new features or functions to systems according to their own needs, or simply increase hardware facilities (server) according to their situation, improving system performance instead of changing the whole platform framework [7].

\section{4 High Security Ttrategy and Friendly Management Mechanism}

Platform should build adequate high security strategy and friendly management mechanism to guarantee user information safety by various methods such as identity authentication, data encryption, access control etc. 


\section{The structure and functional analysis of network teaching platform based grid}

\subsection{The Structure of Platform}

As the most influential grid architecture, OGSA (Open Grid Services Architecture) provides an serviceoriented architecture for the design of network teaching platform based on grid [2]. In network teaching platform, what we need solve is sharing and cooperation of distributed information resources, therefore, we need to establish a teaching platform based on grid according to OGSA. There are five modules that constitute a network teaching platform based on grid. They are grid hardware layer module, resource layer module, grid operating system layer module, service layer module and application layer module. The structure of teaching platform based on OGSA is shown in Fig. 2. GHLM refers to grid hardware layer module, RLM refers to resource layer module, GOSLM refers to grid operating system layer module, SLM refers to service layer module, ALM refers to application layer module.

\subsection{The Analysis of Module Function}

\section{1) Grid hardware layer module}

Grid hardware module is the base of the whole platform, which includes all kinds of heterogeneous resources distributed in different places, including various computers, large storage equipment, database, high performance computer clusters and valuable scientific instruments and equipment.

\section{2) Resource layer module}

This module deals with the data from data grid and the Calculations from computing grid to form information resources, and provide them to service grid.

\section{3) Grid operating system layer module}

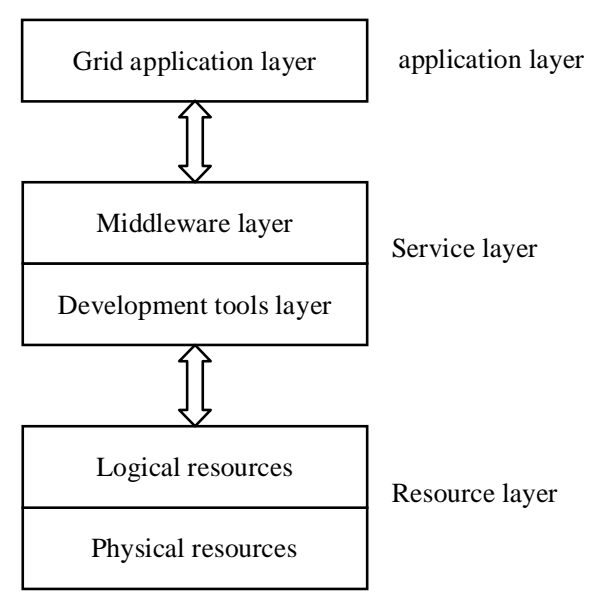

Figure 2. The structure of teaching platform based on OGSA

Grid operating system is a kind of system software to complete resources management, data management, and provide protocol processing application programming interface [8]. It is developed on the basis of grid hardware, and is also called middleware. Grid operating system is the core of grid teaching platform. 


\section{4) Service layer module}

Service layer module is a platform for managing and treating integrated information, and provides various services for the comprehensive use of resources.

\section{5) Application layer module}

Grid application for users provides an application-oriented environment of integrated information. Application layer is the core of grid teaching platform. It is designed to interface with various application fields directly. In this module, we can design different function sub grids to meet the needs of users, for example: E-learning grid, virtual community grid, safety control grid and Tele-immersion [7].

\section{6. conclusion}

With the continuous development and improvement of grid technology, many campus grid construction projects will be constructed making it possible to apply grid technology to network teaching. In the near future, network teaching based on grid will usher in a new era of network teaching.

The application of grid technology to network teaching will eliminate and solve the disadvantages and problems which exist in traditional network teaching, however, grid technology is a very complicated engineering system, and designing a perfect grid teaching platform to make full use of the advantages of grid technology is still on the horizon.

\section{References}

[1] L. Foster and C. Kesselman, Grid Computing (second edition). Beijing: China Machine Press, April 2005.

[2] L. Foster, C. Kesselman, J. Nick and S. Tuecke, "Grid services for distributed systems integration," IEEE Computer. vol. 35(6), pp. 37-46, 2002.

[3] Guo Yu, "On the Grid in the field of education and analysis of the application," Computer Knowledge and Technology. vol. 2(18) , pp. 1684-1688, 2008. (in Chinese)

[4] Du Zhihui, Chen Yu and Liu Peng, Grid Computing. Tsinghua University Press, pp. 12- 15, 2002. (in Chinese)

[5] Peng Yahong and Liu Bin , “Talking about grid technology [EB/OL]," http://www.net130.com/2004/12-2/101835.html. (in Chinese)

[6] Sun Chuanfu, "Talkinging about the problems existing in university network teaching," Sci-tech information develoopment\&economy. vol. 17(22) , pp. 257-258, 2007. (in Chinese)

[7] Chen Ping, "Research and design of university teaching platform based on Grid mode," Development and application of computer. vol. 19(7), pp. 41-43, 2006. (in Chinese)

[8] Wang Fei and Zheng Xiaowei, "The research and design of network teaching system based on grid technology,” Journal of Liaoning Normal University (Natural Science Edition). vol. 32(1) , pp. 61-63, 2009. (in Chinese) 